\title{
Acute kidney injury in elderly intensive care patients from a developing country: clinical features and outcome
}

This article was published in the following Dove Press journal: International Journal of Nephrology and Renovascular Disease 3 February 2017

Number of times this article has been viewed

\section{Laís Gabriela Yokota \\ Beatriz Motta Sampaio \\ Erica Rocha \\ André Luís Balbi \\ Daniela Ponce}

Internal Medicine Department, University São Paulo State-UNESP, Distrito de Rubiao Junior, Botucatu, Sao Paulo, Brazil
Correspondence: Daniela Ponce University São Paulo State-UNESP, Av. Prof. Mário Rubens Guimarães Montenegro, Botucatu, Sao Paulo |86|8687, Brazil Tel +55 I497625806 Email dponce@fmb.unesp.br
Aim: The elderly are at high risk of acute kidney injury (AKI) because of structural and functional degeneration over time and with the aging of the population, the demand for intensive care unit (ICU) admission for older patients has risen recently. However, data from developing countries are scarce. This study aimed to describe the incidence of AKI in elderly patients admitted to ICU from a developing country, to determine the most frequent etiologies for renal impairment and identify its risk factors and outcome.

Methods: All patients admitted to the ICU at a Brazilian teaching hospital for 12 consecutive months were followed prospectively from the time of admission until ICU discharge. Elderly was defined as aged $>60$ years and AKI was defined according to the Kidney Disease Improving Global Outcomes 2012 criteria. Multivariable logistic regression was used to adjust confounding and selection bias.

Results: Two hundred elderly patients were included in the study. AKI incidence was $27 \%$ and the main etiology was sepsis $(48.1 \%$ ). At logistic regression, baseline creatinine (odds ratio $[\mathrm{OR}]=5.17, p<0.0001$ ), Acute Physiology and Chronic Health Evaluation (APACHE) II $(\mathrm{OR}=1.20, p<0.0001)$, sepsis $(\mathrm{OR}=2.96, p<0.0001)$, and longer ICU stay $(\mathrm{OR}=1.68, p<0.0001)$ were associated with $\mathrm{AKI}$ in elderly patients. Baseline creatinine $(\mathrm{OR}=1.97, p=0.018), \mathrm{APACHE}$ II $(\mathrm{OR}=1.29, p<0.0001)$, sepsis $(\mathrm{OR}=1.87, p<0.0001)$, and AKI severity $(\mathrm{OR}=2.57, p=0.027)$ were identified as predictors of death.

Conclusion: AKI was frequent in elderly patients admitted to ICU from a developing country, and it was identified as a risk factor for death. Sepsis was an important risk factor for both AKI and mortality, similar to developed countries and in younger populations.

Keywords: acute kidney injury, elderly, intensive care, mortality

\section{Introduction}

Acute kidney injury (AKI) is a common and important occurrence in intensive care units (ICU), ${ }^{1}$ and several studies support the statement that the elderly patient is at the highest risk of AKI, which is associated with considerable mortality $(15 \%-40 \%) .^{2}$ The elderly population is growing globally and, consequently, it has been accompanied by an increase in the incidence of AKI. A US study showed that the incidence of AKI increased progressively with age, from 13.6 per 1000 patient-years in those aged 66-69 years to 46.9 per 1000 patient-years in those aged $\geq 85$ years. $^{3}$

The higher incidence of AKI in elderly persons can be attributed to the comorbidities, need for procedures, drugs that function as nephrotoxins, and to the kidney that undergoes age-dependent structural and functional alterations over time., ${ }^{4,5}$ 
Few studies in the published literature attribute etiology to AKI and discriminate between acute tubular necrosis (ATN) and prerenal AKI. ${ }^{6-9}$ They have estimated that $40 \%$ of AKI in the elderly is due to ATN and $30 \%$ due to prerenal causes. ${ }^{10}$ Approximately one-quarter of AKI in elderly patients is due to obstruction. ${ }^{6,11}$ However, most of these studies were performed in developed countries and data from developing countries are scarce. Moreover, patients were not only in ICU but also in general wards of hospital. Given the paucity of studies in this important area, more studies on AKI in elderly patients and its effect on clinical outcomes are necessary.

In this study, we hypothesize that the incidence of AKI in elderly critically ill patients admitted to ICU from a developing country is high and its etiologies are similar to those from developed countries, and that its risk factors and outcome are not discernibly different from AKI in younger populations.

\section{Materials and methods}

This was an observational study. All patients admitted to the general ICU at Botucatu School of Medicine, Sao Paulo, Brazil (University of Sao Paulo State-UNESP) were followed prospectively from the time of admission of ICU through ICU discharge. Nephrology fellows visited the ICU daily from October 2014 to September 2015 and collected data on all elderly patients admitted in this ICU.

Elderly was defined according to Brazilian law ( $>60$ years old) and AKI was defined according to the Kidney Disease Improving Global Outcomes (KDIGO) criteria based on the increase of serum creatinine or decrease of urine output. ${ }^{12} \mathrm{We}$ excluded chronic kidney disease (CKD) patients stages 4 and 5 (clearance de creatinine $<30 \mathrm{~mL} / \mathrm{min}$ ), kidney transplantation, ICU stay $<24 \mathrm{~h}$, and patients already admitted in ICU with AKI. Baseline glomerular filtration rate was estimated using the modification of the diet in renal disease equation. ${ }^{13}$ Baseline creatinine was defined as the lowest serum creatinine value in the last 6 months before AKI or, for those without this measurement, the lowest value achieved during hospitalization in the absence of dialysis. ${ }^{14}$

Variables previously reported to be associated with AKI in other populations and those with a plausible association with AKI were included in the risk factor analysis. Baseline data, including demographics, medical history, and severity, were collected prospectively on each patient by review of the medical record. Key risk factors analyzed are displayed in Table 1. Laboratory characteristics were recorded for the first ICU day and vital signs, hemodynamic and laboratory data were recorded each day during ICU stay. Renal function was assessed daily based on creatinine levels and urine output. Serial Acute Physiology and Chronic Health Evaluation
Table I Elderly patients demographics and clinical characteristics according to the presence of AKI $(n=200)$

\begin{tabular}{|c|c|c|c|c|}
\hline Characteristics & $\begin{array}{l}\text { Total } \\
(\mathbf{N}=\mathbf{2 0 0})\end{array}$ & AKI $(N=54)$ & $\begin{array}{l}\text { Non-AKI } \\
(\mathrm{N}=146)\end{array}$ & p-Value \\
\hline Age (years) & $70.94 \pm 7.86$ & $72.4 \pm 7.60$ & $70.4 \pm 7.91$ & 0.138 \\
\hline $\begin{array}{l}\text { Baseline creatinine } \\
(\mathrm{mg} / \mathrm{dL})\end{array}$ & $1.17 \pm 0.7$ & $1.54 \pm 0.99$ & $0.99 \pm 0.4$ & $<0.001$ \\
\hline Male (\%) & $107(53.5)$ & $28(51.8)$ & $79(54.1)$ & 0.94 \\
\hline Heart rate (bpm) & $85.83 \pm 21.91$ & $96 \pm 22.4$ & $82.1 \pm 20.6$ & $<0.001$ \\
\hline initial SBP (mmHg) & $|32.7 \pm 3| . \mid$ & $106.6 \pm 29.2$ & $143.1 \pm 35.2$ & $<0.01$ \\
\hline Hypertension (\%) & $|4|(70.5)$ & $39(72.2)$ & $102(69.9)$ & 0.74 \\
\hline Diabetes (\%) & $78(39)$ & $26(48.1)$ & $52(32.2)$ & 0.08 \\
\hline $\begin{array}{l}\text { Cardiovascular } \\
\text { disease (\%) }\end{array}$ & $65(32.5)$ & $22(40.7)$ & $43(29.5)$ & 0.08 \\
\hline \multicolumn{5}{|c|}{ Cause of ICU admission (\%) } \\
\hline $\begin{array}{l}\text { Acute } \\
\text { cardiovascular } \\
\text { event* }\end{array}$ & $101(50.5)$ & $19(35.2)$ & $82(56.2)$ & 0.003 \\
\hline Sepsis & $69(34.5)$ & $26(48.1)$ & $43(29.4)$ & $<0.001$ \\
\hline Surgery & $30(15)$ & $9(16.7)$ & $21(13.5)$ & 0.42 \\
\hline APACHE II & $12.73 \pm 5.97$ & $17.5 \pm 7.5$ & $11.3 \pm 4.2$ & $<0.001$ \\
\hline ICU stay (days) & $6.88 \pm 7.91$ & $1 \mathrm{I} .4 \pm 5.4$ & $5.2 \pm 2.1$ & $<0.001$ \\
\hline Temperature $\left({ }^{\circ} \mathrm{C}\right)$ & $36.42 \pm 1.88$ & $37.4 I \pm 1.02$ & $36.14 \pm 1.10$ & 0.004 \\
\hline $\begin{array}{l}\text { Respiratory rate } \\
\text { (breaths per minute) }\end{array}$ & $19.29 \pm 8.81$ & $|8.8 \pm 5|$. & $19.1 \pm 9.6$ & 0.55 \\
\hline $\begin{array}{l}\text { Noradrenaline } \\
\text { use (\%) }\end{array}$ & $52(26)$ & $16(29.6)$ & $36(24.6)$ & 0.51 \\
\hline $\begin{array}{l}\text { Mechanical } \\
\text { ventilation (\%) }\end{array}$ & $66(33)$ & $20(37.0)$ & $46(31.5)$ & 0.58 \\
\hline Death (\%) & $49(24.5)$ & $26(48.1)$ & $23(15.7)$ & $<0.001$ \\
\hline
\end{tabular}

Note: *Acute vascular event (stroke and acute myocardial infarction). Abbreviations: AKI, acute kidney injury; APACHE, Acute Physiology and Chronic Health Evaluation; bpm, beats per minute; ICU, intensive care unit; SBP, systolic blood pressure.

(APACHE) II score was computed on the first ICU day, and AKI was classified according to KDIGO stage after 7 days from AKI diagnosis. ${ }^{7}$ All elderly patients were divided into groups AKI and non-AKI and survivors and nonsurvivors.

Etiology of AKI was classified as prerenal, renal (ischemic and nephrotoxic ATN, associated with septic or mix disorder), and postrenal. Diagnosis was based on clinical history, results of physical examination, relevant blood tests, urinalysis, and the findings on renal ultrasonography.

The primary outcome was to identify risk factors for AKI and in-ICU mortality. Informed consent was obtained from study participants or their legal caregiver. The study was approved by the ethics committee of Botucatu School of Medicine on September 11, 2014 (protocol number 33811114.0.0000.5411).

\section{Statistical analysis}

Continuous variables were expressed as means \pm standard deviation (SD) or medians and compared using the Student's $t$-test or the Wilcoxon rank sum test, as appropriate. 
Categorical variables were expressed as proportions and compared with the chi-squared test. Variables with significant univariate associations were candidates for multivariable analysis. Logistic regression was performed using backward variable selection, with the exit criteria set at $p<0.20$. Variables not selected by the automated procedure were added back into models individually to evaluate for residual confounding and covariate, and propensity score adjustments were used to adjust for baseline differences. $p$-values $<0.05$ were considered significant.

Standardized risks were determined for each variable in the model using post-estimation marginal analysis. This method allows an estimation of the absolute risk of AKI in the presence or absence of a single characteristic, holding the other covariates in the model at the average values of the cohort. For mortality analyses, the unadjusted associations of AKI with death at discharge were evaluated using Pearson's chi-squared test. Associations of baseline variables with mortality were also determined to identify possible confounders (defined as in the primary multivariable model). Multivariable logistic regression was then used to adjust the effect of AKIN on death for these confounders. Statistical analyses were conducted using SPSS 13.0 (May, 2010).

Two hundred elderly patients were included in the final analysis (Figure 1). AKI developed in 54 patients (27\%) and most of the AKI episodes were diagnosed by the increase in serum creatinine $(77.8 \%)$ and only $22.2 \%$ was diagnosed by urine output criteria. Among AKI patients, most of the patients were classified as AKI stage 1 (42.5\%), followed by stage $3(38.9 \%)$. The main etiology was ATN associated with sepsis (48.1\%). There was not obstructive AKI etiology.
A comparison of baseline characteristics between elderly who did and did not develop AKI is shown in Table 1 . AKI group had higher APACHE II $(17.5 \pm 7.5 \times 11.3 \pm 4.2, p<0.001)$, sepsis as the main cause of ICU admission $(48.1 \% \times 29.4 \%$, $p<0.001)$, higher baseline creatinine $(1.54 \pm 0.99 \times 0.99 \pm 0.4$, $p<0.001)$, higher temperature $(37.41 \pm 1.02$ vs. $36.14 \pm 1.10$, $p=0.004)$ and heart rate $(96 \pm 22.4 \times 82.1 \pm 20.6, p<0.001)$, longer ICU stay (11.4 \pm 5.4 vs. $5.2 \pm 2.1, p<0.001)$, and higher mortality rate $(48.1 \%$ vs. $15.7 \%, p<0.0001)$.

The groups were similar in age, gender, diabetes, hypertension, cardiovascular disease, need for noradrenaline use, and the need for mechanical ventilation at ICU admission.

The general mortality rate in elderly admitted to ICU was $24.5 \%$. Nonsurvivors patients had higher APACHE II $(19.1 \pm 6.62 \times 11.0 \pm 4.25, p<0.001)$, more sepsis $(61.2 \% \times 26.9 \%, p<0.0001)$, higher baseline creatinine $(1.43 \pm 0.95 \times 1.07 \pm 0.56, p=0.007)$, more AKI stage $3(72.2 \%$ vs. $27.8 \%, p<0.001)$, less prerenal AKI ( $8.2 \%$ vs. $58.3 \%$, $p<0.001)$, longer ICU stay (11.3 \pm 5.8 vs. $5.9 \pm 2.8, p<0.001)$, and higher need for mechanical ventilation as well as need for noradrenaline use ( $17.2 \%$ vs. $42.8 \%, p=0.02$ and $46.9 \%$ vs. $20 \%, p<0.0001$, respectively). Survivors and nonsurvivors patients were similar in age, gender, diabetes, hypertension, cardiovascular disease, and AKI incidence (Table 2).

Logistic regression analysis identified as factors associated with AKI higher baseline creatinine (odds ratio $[\mathrm{OR}]=5.17, p<0.0001)$ and APACHE II $(\mathrm{OR}=1.20, p<0.001)$, sepsis $(\mathrm{OR}=2.96, p<0.0001)$ and longer ICU stay $(\mathrm{OR}=1.68$, $p<0.001)$ as shown in Table 3.

Baseline creatinine $(\mathrm{OR}=1.97, p=0.018)$, APACHE II $(\mathrm{OR}=1.29, p<0.0001)$, sepsis $(\mathrm{OR}=1.87, p<0.0001)$, and $\mathrm{AKI}$

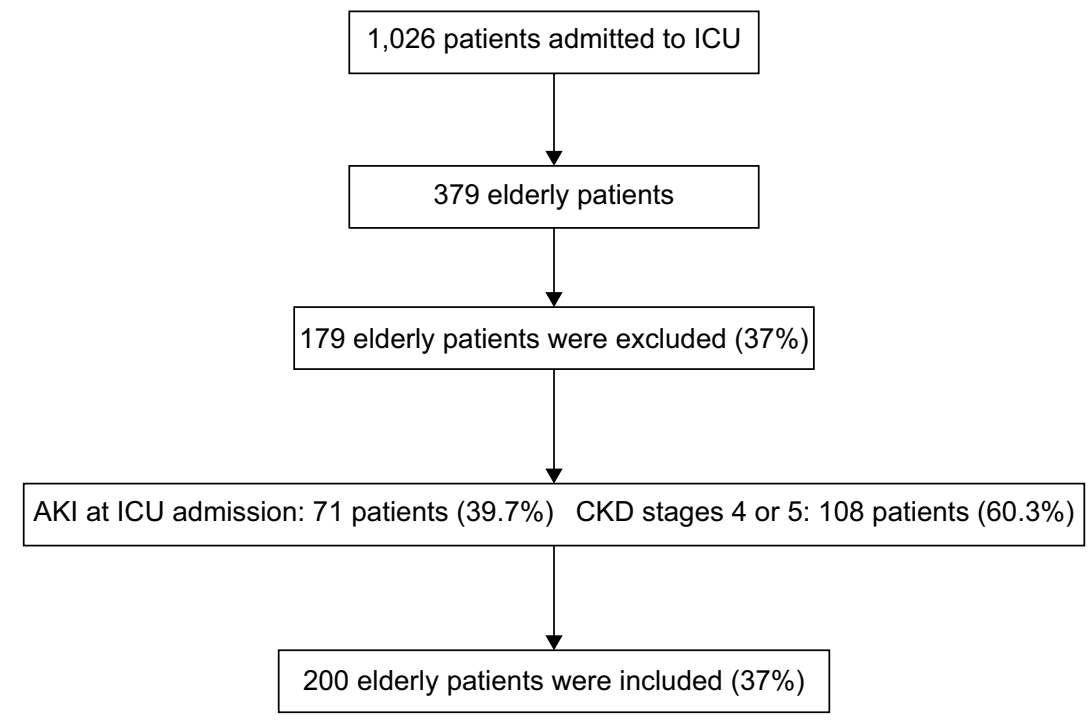

Figure I Screening and enrollment.

Abbreviations: AKI, acute kidney injury; CKD, chronic kidney disease; ICU, intensive care unit. 
Table 2 Elderly patients' demographics and clinical characteristics according to outcome (AKI; $n=200)$

\begin{tabular}{|c|c|c|c|}
\hline Characteristics & $\begin{array}{l}\text { Survivors } \\
(\mathrm{N}=\mid \mathbf{4 5})\end{array}$ & $\begin{array}{l}\text { Nonsurvivors } \\
(\mathbf{N}=49)\end{array}$ & $p$-Value \\
\hline Age (years) & $70.7 \pm 7.78$ & $71.9 \pm 8.19$ & 0.34 \\
\hline Baseline creatinine $(\mathrm{mg} / \mathrm{dL})$ & $1.07 \pm 0.56$ & $1.43 \pm 0.95$ & 0.007 \\
\hline Male (\%) & $75(5 \mathrm{I} .7)$ & $26(53)$ & 0.94 \\
\hline AKI (\%) & $36(24.7)$ & $18(36.7)$ & 0.08 \\
\hline \multicolumn{4}{|l|}{ KDIGO } \\
\hline Stage I & $22(6 I .1)$ & $2(11.1)$ & $<0.001$ \\
\hline Stage 2 & $6(16.7)$ & $3(6.1)$ & 0.43 \\
\hline Stage 3 & $8(22.2)$ & $13(72.2)$ & $<0.001$ \\
\hline \multicolumn{4}{|l|}{ AKI etiology (\%) } \\
\hline Prerenal & $21(58.3)$ & $4(8.2)$ & $<0.01$ \\
\hline Renal & $15(4 \mid .7)$ & 14 (77.8) & $<0.01$ \\
\hline Diabetes (\%) & $26(48.1)$ & $52(32.2)$ & 0.08 \\
\hline Cardiovascular disease (\%) & $22(40.7)$ & $43(29.5)$ & 0.07 \\
\hline Sepsis (\%) & $39(26.9)$ & $30(6 \mid .2)$ & $<0.00$ I \\
\hline APACHE II & $11.0 \pm 4.25$ & $19.1 \pm 6.62$ & $<0.001$ \\
\hline ICU stay (days) & $5.9 \pm 2.8$ & $11.3 \pm 5.8$ & $<0.001$ \\
\hline Noradrenaline use (\%) & $29(20)$ & $23(46.9)$ & $<0.001$ \\
\hline Mechanical ventilation (\%) & $25(17.2)$ & $21(42.8)$ & 0.02 \\
\hline
\end{tabular}

Abbreviations: AKI, acute kidney injury; APACHE, Acute Physiology and Chronic Health Evaluation; ICU, intensive care unit; KDIGO, Kidney Disease Improving Global Outcomes.

stage $3(\mathrm{OR}=2.57, p=0.027)$ were identified as the predictors of death in logistic regression analysis (Table 3 ).

Adjusting for variables such as age, sepsis, and APACHE II, there was no significant change in the association between AKI stage 3 and mortality (Figure 2). Given this finding and the limited number of deaths, only those three variables were included in the multivariable model to avoid over fitting.

\section{Discussion}

This study of elderly patients admitted to the ICU from a Brazilian teaching hospital indicates that $\mathrm{AKI}$ is very frequent and suggest that AKI has an important impact on in-hospital mortality of geriatric patients.

The incidence of AKI is increasing over time and is most common in elderly individuals. This is due to many reasons, including elderly are more likely to have renal structural decline and multiple comorbidities. ${ }^{3,8,9,12,14,15}$ Previous studies reported the incidence of AKI among geriatric patients ranged from $22 \%$ to $40 \%$, with most patients having stage 1 disease. ${ }^{3-5,8,9,12} \mathrm{We}$ found that $27 \%$ of geriatric patients had AKI and most of them had AKI stage 1, in agreement with the literature. ${ }^{15,16}$ The in-ICU mortality of geriatric AKI patients $(48.1 \%)$ was higher than the other studies (16\%-40\%). ${ }^{3-5,16}$ However, these studies also included elderly patients not only in ICUs but also in general wards of the hospital.
Table 3 Logistic regression for AKI and death risk $(n=200)$

\begin{tabular}{lll}
\hline & OR (95\% CI) & p-Value \\
\hline AKI & $1.03(0.99-1.07)$ & 0.14 \\
Age & $1.18(0.94-1.36)$ & 0.17 \\
Cardiovascular disease & $5.17(2.24-11.96)$ & $<0.00$ I \\
Baseline creatinine & $1.20(1.13-2.38)$ & $<0.00$ I \\
APACHE II & $1.16(0.98-2.20)$ & 0.08 \\
Diabetes & $1.21(0.92-1.67)$ & 0.11 \\
Temperature & $2.96(1.86-4.99)$ & $<0.001$ \\
Sepsis & $1.68(1.21-3.19)$ & $<0.001$ \\
Longer ICU stay & & \\
Death & $1.03(0.98-1.17)$ & 0.19 \\
Age & $1.08(0.96-1.26)$ & 0.11 \\
Cardiovascular disease & $1.97(1.54-4.85)$ & 0.018 \\
Baseline creatinine & $1.29(1.73-3.88)$ & $<0.001$ \\
APACHE II & $1.06(0.98-2.01)$ & 0.09 \\
Diabetes & $1.21(0.99-1.67)$ & 0.11 \\
AKI & $2.57(1.14-3.09)$ & 0.027 \\
AKI stage 3 & $1.87(1.76-3.19)$ & $<0.00$ I \\
Sepsis & $1.26(0.99-1.29)$ & 0.09 \\
Longer ICU stay &
\end{tabular}

Abbreviations: AKI, acute kidney injury; APACHE, Acute Physiology and Chronic Health Evaluation; $\mathrm{Cl}$, confidence interval; ICU, intensive care unit; OR, odds ratio.

In this study, the risk factors for AKI in elderly patients were higher APACHE II, presence of sepsis, and higher baseline creatinine. Our findings are consistent with the previous studies performed in ICU in younger populations that have reported that $\mathrm{AKI}$ is more frequently observed in severe and septic patients. $1,2,17,18$

There are few studies on AKI in elderly patients admitted to ICU from developing countries. In India, researchers also found that underlying chronic illness, presence of cardiac failure, and sepsis were associated with AKI and poor outcome among the elderly population in a hospital-based study. ${ }^{19}$ Researchers have shown that chronic kidney patients have chronic reduced glomerular filtration rates, leading to higher risk of AKI in concomitant diseases, such as heart failure, sepsis, ischemic renal disease, and obstructive uropathy., ${ }^{5,20}$

In this study, most of the elderly AKI patients presented one or more concomitant diseases, the most frequent were cardiovascular diseases, diabetes mellitus, and hypertension. These comorbidities were not identified as risk factor for AKI, probably because they were so frequent in studied population.

Sepsis was the main cause of elderly patients with AKI (48.1\%). This is similar to the studies of younger AKI patients in ICU. However, it does not agree with previous studies on general elderly patients with AKI, which is frequently of multifactorial origin and often with an atypical presentation, which combines the characteristics of prerenal azotemia, ATN, and obstructive AKI. ${ }^{21}$ According to the literature, 


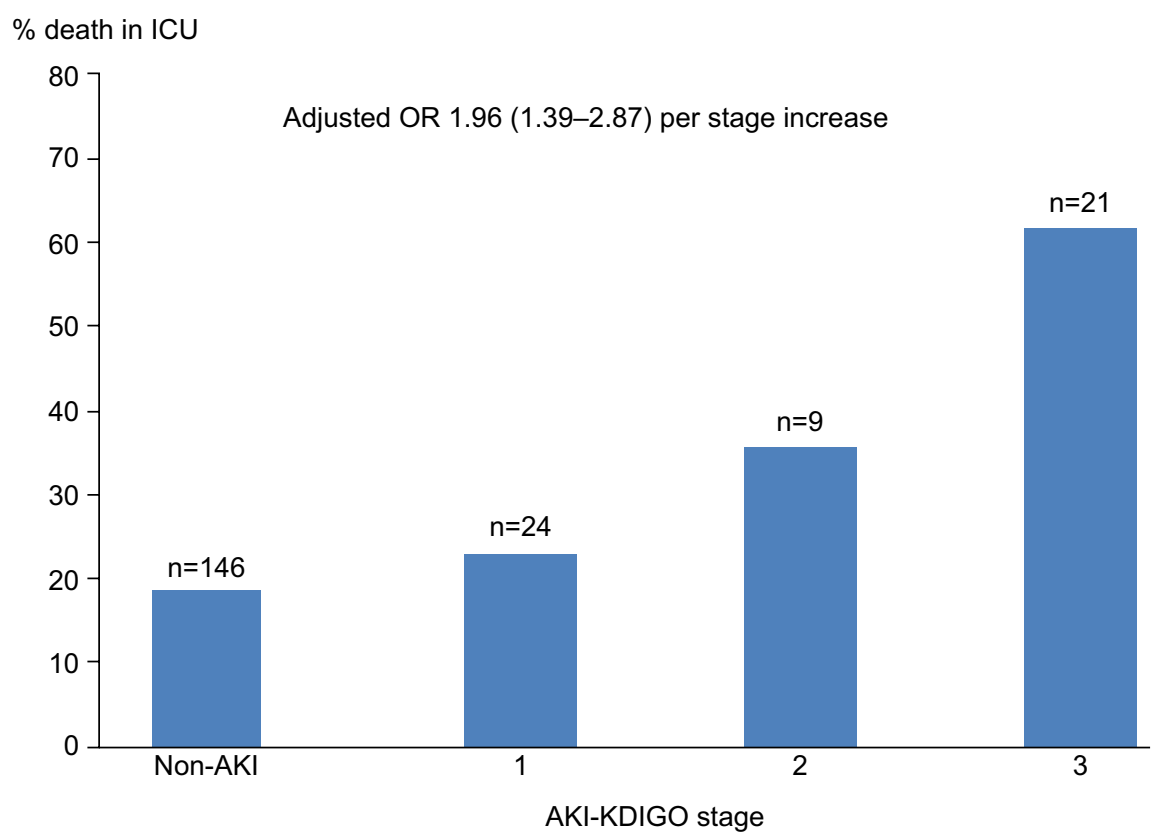

Figure 2 In-ICU, mortality of geriatric patients without AKI and with stages I, 2, and 3 AKI. Numbers at the top of each bar represent the total number of patients for each stage.

Note: OR for death was adjusted for age and APACHE II score.

Abbreviations: AKI, acute kidney injury; APACHE, Acute Physiology and Chronic Health Evaluation; ICU, intensive care unit; KDIGO, Kidney Disease Improving Global Outcomes; OR, odds ratio.

sepsis is a well-known risk factor for the development of AKI, occurring in $\sim 19 \%$ of patients with sepsis, $23 \%$ of patients with severe sepsis, and $51 \%$ of patients with septic shock. ${ }^{22,23}$ AKI associated with sepsis remains a major challenge in ICU due to its common occurrence, the high costs of treatment, and the unacceptable high mortality. Although physicians have a critical role in the management of patients with severe sepsis and septic shock, there are few studies about the incidence and outcome of elderly patients with sepsis in ICU. ${ }^{24}$ Whether the septic elderly AKI is different from younger patients needs to be further studied and clarified.

It is widely recognized that AKI leads to significant impact on morbidity, increased length of stay, hospital costs, and poorer outcomes among critically ill patients, but these findings are scarce in developing countries. ${ }^{1-5,8,9,12}$ Overall inhospital mortality rates among critically ill patients are high, exceeding $50 \%$ and reaching $80 \%$, depending on the clinical conditions, comorbidities, and need for renal replacement therapy. However, few studies have examined the association between AKI and in-ICU mortality in elderly patients.

In accordance with other studies, we observed that the mortality rate of the elderly AKI patients was $48.1 \%$ and higher than that of the elderly patients without AKI, which was $15.7 \%$. Also, in a prospective study of elderly AKI patients (aged $>60$ years) performed in Brazil, it was reported that the mortality rate of the elderly AKI patients was $54 \%{ }^{25}$ Kohli et $\mathrm{al}^{6}$, in a prospective study, found a high mortality rate of $61 \%$ in the elderly patients with AKI (aged $>60$ years) in a tertiary care center of India.

In this study, baseline creatinine, APACHE II, sepsis, and AKI severity (stage 3 ) were identified as predictors of death in logistic regression analysis. It is similar to previous results reported in the literature.

APACHE II, SAPS II scores, and SOFA scores are considered predictors for mortality in the elderly AKI patients. ${ }^{26}$ Previous studies also found that SOFA score of AKI patients had good discriminative power for ICU and hospital mortality. ${ }^{27}$ In the study by Varricatt et al, ${ }^{28}$ statistically significant correlation was found between Liano (also referred to as ATN-ISI) score and mortality of AKI patients $(p<0.001)$.

Sural et $\mathrm{al}^{29}$ showed that the mortality rate increased from $39 \%$ to $100 \%$ when the number of failed organs increased from two to five in elderly AKI patients. Kohli et $\mathrm{al}^{6}$ showed that sepsis and severity of illness were independent predictors of mortality in a geriatric population.

Researchers believed that the high mortality rate in AKI is not explained by the underlying conditions alone. AKI appears to increase the risk of developing severe nonrenal complications that lead to death and should not be regarded as a treatable complication of serious illness. ${ }^{30}$ 
In other previous study, researchers found older patients and patients with CKD, disease severity, and admission diagnoses in the infectious disease, respiratory, gastrointestinal, and malignancy had an increased risk of death. ${ }^{31}$

In fact, elderly patients are prone to complicating sepsis, AKI, and others diseases. And because of the old age, elderly septic AKI patients are easy to develop multiple organs dysfunction system (MODS), which will increase the mortality rate. Therefore, it is important to pay more attention to the treatment of sepsis and concomitant diseases of elderly AKI patients and prevent them from developing dysfunctions of other organs.

Recently, the KDIGO criteria were developed to standardize the diagnosis of AKI and it gives a level of classification of AKI severity. ${ }^{7}$ According to the previous literature, this study also showed significant difference in AKI stage between the elderly nonsurvivors AKI patients and the elderly survivors AKI patients.

Our study has its strength and weakness. It is among the few reports that evaluated the incidence and risk of AKI using new criteria for it in elderly patients admitted to ICU from developing countries. A high incidence of AKI among elderly AKI patients was detected and sepsis, higher APACHE II, and CKD were identified as risk factors for AKI, serving as an explanation for the mechanisms between AKI and its negative impact on short-term mortality. However, the limitation lies in its focus on geriatric patients admitted to ICU, so that our findings might not be readily applicable to elderly AKI patients in general. Furthermore, it was a single-center observational study and the population size was relatively small.

Finally, we concluded that the presence of AKI at admission of geriatric patients is associated with higher risk of inICU mortality, specifically in septic patients. Moreover, the risk of death was greater for patients with more severe AKI, suggesting that more careful approaches to kidney protection are required in the management of older-aged patients with sepsis. Further studies that examine larger populations are needed to confirm our findings.

\section{Acknowledgments}

Financial support was provided by Fundação de Amparo a pesquisa do Estado de São Paulo (FAPESP) grants. LGY received grant from FAPESP.

\section{Authors' contributions}

All authors contributed toward data analysis, drafting and revising the paper and agree to be accountable for all aspects of the work.

\section{Disclosure}

The authors report no conflicts of interest in this work.

\section{References}

1. Sileanu FE, Murugan R, Lucko N et al. AKI in low-risk versus high-risk patients in intensive care. Clin JAm Soc Nephrol. 2015;10(2):187-196.

2. Coca, S.G. Acute kidney injury in elderly persons. Am J Kidney Dis. 2010;56(1):122-131.

3. Rosner ACMH, Cruz DN, Ronco C. Acute kidney injury in elderly intensive care patients: a review. Intensive Care Med. 2010;36(9):1454-1464.

4. USRDS Annual Report. Acute kidney injury. Am J Kidney Dis. 2013; 61:e97-e108.

5. Pascual J, Liano F. Causes and prognosis of acute renal failure in the very old. Madrid Acute Renal Failure Study Group. J Am Geriatr Soc. 1998;46(6):721-725.

6. Kohli HS, Bhat A, Aravindan SudK, Jha V, Gupta KL, Sakhuja V. Spectrum of renal failure in elderly patients. Int Urol Nephrol. 2006; 38(3-4):759-765.

7. Kidney Disease: Improving Global Outcomes (KDIGO) Acute Kidney Injury Work Group. KDIGO clinical practice guideline for acute kidney injury. Kidney Int Suppl. 2012;2:1-138.

8. Medeiros P, Nga HS, Menezes P, Bridi R, Balbi AL, Ponce D. Acute kidney injury in septic patients admitted to emergency clinical room: risk factors and outcome. Clin Exp Nephrol. 2015;19(5):859-866.

9. Chao CT, Lin YF, Tsai HB, Wu VC, Ko WJ. Acute kidney injury network staging in geriatric postoperative acute kidney injury patients: shortcomings and improvements. J Am Coll Surg. 2013;217(2):240-250.

10. Baraldi A, Ballestri $M$, Rapana R et al. Acute renal failure of medical type in an elderly population. Nephrol Dial Transplant. 1998;13(Suppl 7):25-29.

11. Lameire N, Matthys E, Vanholder R, et al. Causes and prognosis of acute renal failure in elderly patients. Nephrol Dial Transplant. 1987;2(5): 316-322.

12. Abdel-Rahman EM, Okusa MD. Effects of aging on renal function and regenerative capacity. Nephron Clin Pract. 2014;127(1-4):15-20.

13. Levey AS, Bosch JP, Lewis JB, Greene T, Rogers N, Roth D. A more accurate method to estimate glomerular filtration rate from serum creatinine: a new prediction equation. Ann Intern Med. 1999;130(6):461-470.

14. Siew ED, Matheny ME, Ikizler TA, et al. Commonly used surrogates for baseline renal function affect the classification and prognosis of acute kidney injury. Kidney Int. 2010;77(6):536-542.

15. Chao C, Wu VC, Lai CF, et al. Advanced age affects the outcomepredictive power of RIFLE classification in geriatric patients with acute kidney injury. Kidney Int. 2012;82(8):920-927.

16. Chao CT, Tsai HB, Wu CY, et al. The severity of initial acute kidney injury at admission of geriatric patients significantly correlates with subsequent in-hospital complications. Sci Rep. 2015;5:13925.

17. Plataki M, Kashani K, Cabello-Garza J, et al. Predictors of acute kidney injury in septic shock patients: an observational cohort study. Clin J Am Soc Nephrol. 2011;6(7):1744-1751.

18. Suh SH, Kim CS, Choi JS, Bae EH, Ma SK, Kim SW. Acute kidney injury in patients with sepsis and septic shock: risk factors and clinical outcomes. Yonsei Med J. 2013;54(4):965-972.

19. Mahajan S, Tiwar S, Bhowmik D, Agarwal SK, Tiwari SC, Dash SC. Factors affecting the outcome of acute renal failure among the elderly population in India: a hospital based study. Int Urol Nephrol. 2006;38(2): 391-396.

20. Gong Y, Zhang F, Ding F, Gu Y. Elderly patients with acute kidney injury (AKI): Clinical features and risk factors for mortality. Arch Gerontol Geriatr. 2012;54(2):e47-e51.

21. Musso CG, Liakopoulos V, Ioannidis I, Eleftheriadis T, Stefanidis I. Acute renal failure in the elderly: particular characteristics. Int Urol Nephrol. 2006;38(3-4):787-793.

22. Silva E, Pedro MA, Sogayar ACB, et al. Brazilian sepsis epidemiological study (BASES study). Crit Care. 2004;8(4):R251-R260.

23. Rangel-Frausto MS, Pitter D, Costigan M, Hwang T, Davis CS, Wenzel RP. The natural history of the systemic inflammatory response syndrome (SIRS): a prospective study. JAMA. 1995;273(2):117-123. 
24. Angus DC,Linde-ZwirbeWT,LidickerJ,ClermontG, CarcilloJ,PinskyMR Epidemiology of severe sepsis in the United States: analysis of incidence, outcome, and associated costs of care. Crit Care Med. 2001; 29(7):1303-1310.

25. Sesso R, Roque A, Vicioso B, Stella S. Prognosis of ARF in hospitalized elderly patients. Am J Kidney Dis. 2004;44(3):410-419.

26. Ahlstrom A, Kuitunen A, Peltonen S, et al. Comparison of 2 acute renal failure severity scores to general scoring systems in the critically ill. Am J Kidney Dis. 2006;48(2):262-268.

27. de Mendonca A, Vincent JL, Suter PM, et al. Acute renal failure in the ICU: risk factors and outcome evaluated by the SOFA score. Intensive Care Med. 2000;26(7):915-921.
28. Varricatt VP, Rau NR, Attur RP, Baig WW. Validation of Liano score in acute renal failure: a prospective study in Indian patients. Clin Exp Nephrol. 2009;13(1):33-37.

29. Sural S, Sharma RK, Singhal MK, et al. Acute renal failure in an intensive care unit in India - prognostic factors and outcome. J Nephrol. 1999;12(6):390-394.

30. Mary VD, Dunser MW, Greil V, et al. Causes of death and determinants of outcome in critically ill patients. Crit Care. 2006;10(6): R154.

31. Chao TC, Tsai HB, Wu CH, Hsu, Lin YF, Chen JS. Cross-sectional study of the association between functional status and acute kidney injury in geriatric patients. BMC Nephrol. 2015;16:186.
The International Journal of Nephrology and Renovascular Disease is an international, peer-reviewed open access journal focusing on the pathophysiology of the kidney and vascular supply. Epidemiology, screening, diagnosis, and treatment interventions are covered as well as basic science, biochemical and immunological studies. The manuscript management system is completely online and includes a very quick and fair peer-review system, which is all easy to use. Visit http://www. dovepress.com/testimonials.php to read real quotes from published authors.

Submit your manuscript here: https:/www.dovepress.com/international-journal-of-nephrology-and-renovascular-disease-journal 